\title{
Article \\ Community-Acquired Pneumonia among Patients with COPD in Spain from 2016 to 2019. Cohort Study Assessing Sex Differences in the Incidence and Outcomes Using Hospital Discharge Data
}

\author{
Javier de Miguel-Diez ${ }^{1}{ }^{(D}$, Marta Lopez-Herranz $^{2, *}$, Valentin Hernandez-Barrera ${ }^{3}$, Jose M. de Miguel-Yanes ${ }^{4} \mathbb{D}$, \\ Napoleon Perez-Farinos ${ }^{5}$ iD, Julia Wärnberg ${ }^{6}{ }^{(D)}$, David Carabantes-Alarcon ${ }^{7} \mathbb{D}$, Rodrigo Jimenez-Garcia ${ }^{7}$ (D) \\ and Ana Lopez-de-Andres ${ }^{7}$ D
}

check for updates

Citation: de Miguel-Diez, J.; LopezHerranz, M.; Hernandez-Barrera, V.; de Miguel-Yanes, J.M.; Perez-Farinos, N.; Wärnberg, J.; Carabantes-Alarcon, D.; Jimenez-Garcia, R.; Lopez-deAndres, A. Community-Acquired Pneumonia among Patients with COPD in Spain from 2016 to 2019. Cohort Study Assessing Sex Differences in the Incidence and Outcomes Using Hospital Discharge Data. J. Clin. Med. 2021, 10, 4889. https://doi.org/10.3390/jcm10214889

Academic Editor: Pierachille Santus

Received: 20 July 2021

Accepted: 22 October 2021

Published: 23 October 2021

Publisher's Note: MDPI stays neutral with regard to jurisdictional claims in published maps and institutional affiliations.

Copyright: (c) 2021 by the authors. Licensee MDPI, Basel, Switzerland. This article is an open access article distributed under the terms and conditions of the Creative Commons Attribution (CC BY) license (https:/ / creativecommons.org/licenses/by/ $4.0 /)$.
1 Respiratory Department, Instituto de Investigación Sanitaria Gregorio Marañón (IiSGM), Hospital General Gregorio Marañón, Universidad Complutense de Madrid, 28040 Madrid, Spain; javier.miguel@salud.madrid.org

2 Nursing Department, Faculty of Nursing, Physiotherapy and Podology, Universidad Complutense de Madrid, 28040 Madrid, Spain

3 Preventive Medicine and Public Health Teaching and Research Unit, Health Sciences Faculty, Universidad Rey Juan Carlos, Alcorcón, 28922 Madrid, Spain; valetin.hernandez@urjc.es

4 Internal Medicine Department, Instituto de Investigación Sanitaria Gregorio Marañón (IiSGM), Hospital General Gregorio Marañón, Universidad Complutense de Madrid, 28040 Madrid, Spain; josemaria.demiguel@salud.madrid.org

5 Instituto de Investigación Biomédica de Málaga (IBIMA), School of Medicine, Universidad de Málaga, 29071 Málaga, Spain; napoleon.perez@uma.es

6 Instituto de Investigación Biomédica de Málaga (IBIMA), School of Health Sciences, Universidad de Málaga, 29071 Málaga, Spain; jwarnberg@uma.es

7 Department of Public Health \& Maternal and Child Health, Faculty of Medicine, Universidad Complutense de Madrid, 28040 Madrid, Spain; dcaraban@ucm.es (D.C.-A.); rodrijim@ucm.es (R.J.-G.); anailo04@ucm.es (A.L.-d.-A.)

* Correspondence: martal11.ucm@gmail.com; Tel.: +34-91-394-1521

Abstract: Background: To describe and analyze the incidence and hospital outcomes of patients admitted with community-acquired pneumonia (CAP) according to Chronic Obstructive Pulmonary Disease (COPD) status and sex in Spanish hospitals from 2016 to 2019. Methods: We conducted a cohort study using national hospital discharge data of all patients $\geq 40$ years with CAP. Results: A total of 500,833 patients (59.0\% men) was identified. Incidence of CAP increased over time. Ageadjusted incidence was 4.42-times higher in COPD patients. In-hospital mortality (IHM) was lower in men and women with COPD than in those without COPD (14.41\% vs. $10.70 \%$ in men; $11.12 \%$ vs. $8.58 \%$. in women; $p<0.001)$. The risk of dying in hospital increased with age, presence of several comorbidities (excluding T2DM that was a protective factor), and need for mechanical ventilation (non-invasive and invasive) during admission, irrespective of sex. Over time, the IHM decreased significantly in men and women with COPD. Men with COPD were significantly more likely to die in hospital than were COPD women (OR 1.13; 95\% CI 1.07-1.21). Conclusions: Incidence of CAP was higher among subjects with COPD, although the effect of COPD was higher in men than in women. By contrast, IHM was lower in COPD patients, but men with COPD were significantly more likely to die in hospital than were COPD women.

Keywords: community-acquired pneumonia; COPD; sex; incidence; in-hospital mortality

\section{Introduction}

Community acquired pneumonia (CAP) is an infection of the pulmonary parenchyma that results in a huge economic and health burden [1]. It is a major cause of hospitalizations, morbidity, and mortality [2]. Chronic obstructive pulmonary disease (COPD) increases the 
susceptibility to CAP [3,4]. COPD is the third leading cause of death worldwide [5]. COPD patients are at increased risk of developing pneumonia [6], which can be related to more pathogen colonization as a consequence of altered immune mechanisms, which results in higher susceptibility for infection [7]. In addition, COPD can be an adverse prognostic factor for patients hospitalized with pneumonia [8]. However, the influence of COPD on mortality related to CAP is inconclusive. Thus, some authors demonstrated that COPD reduced [9], increased [10], or did not affect mortality [11].

It has been previously reported that sex is a variable associated with the incidence and outcomes of CAP [12]. In fact, CAP has been described to be more severe in men than in women, leading to higher mortality in the former, especially in older age [12]. However, its effect in patients with CAP according to COPD status is unknown. Identifying sex differences in CAP patients with and without COPD can be useful to guide prognostication of patients and their management.

Large population-based hospital discharge databases can be useful to obtain a "realworld" perspective of the influence of COPD on CAP and to assess sex-differences in this possible association [13].

The objectives of this investigation are (1) to assess if suffering COPD affects the incidence of CAP in the Spanish population from 2016 to 2019; (2) to compare the clinical profile (comorbidities and procedures) and the mortality during the hospitalization between those with and without COPD and for women and men separately; and (3) to identify which of the study variables are independently associated with in-hospital mortality (IHM) for patients hospitalized with CAP according to sex and the presence of COPD.

\section{Materials and Methods}

A cohort study was carried out based on hospital discharge reports collected through the Hospital Discharge Records of the Spanish National Health System (HDRSNHS). According to the Spanish legislation, it is mandatory that all public and private hospitals send data on their discharges to the Ministry of Health every month. The study period was from 1 January 2016 to 31 December 2019. The discharge records are coded based on the International Classification of Disease, Tenth Revision (ICD-10). Details on HDRSNHS are available online [14].

The inclusion criteria were (1) patients aged $\geq 40$ years; (2) patients discharged with a primary diagnosis of CAP, using the specific ICD-10 codes described in Table S1, and with a "Present on Admission" (POA) indicator of "Yes", and (3) patients discharged with a secondary diagnosis of CAP (codes shown in Table S1) and with the POA indicator of "Yes". We excluded those patients with "Not specified sex" and those lacking information regarding their length of hospital stay. The flowchart of the study sample selection can be found in Figure S1.

The population was divided according to sex and to COPD status. Subjects with a diagnosis code for COPD (J44.0, J44.1, J44.9) in any diagnosis field were classified as having COPD.

The main study variables were trends from the year 2016 to 2019 in the incidence of CAP, the length of hospital stay (LOHS), and the IHM among women and men with and without COPD. We also analyzed comorbidities and therapeutic procedures.

To estimate the incidences for the years analyzed, we obtained data from the Spanish National Institute of Statistics (SNIS) [15] and the Spanish National Health Survey for the year 2017 (SNHS2017) [16]. Combining these two databases, we calculated the number of women and men aged 40 years or over who suffered COPD in each of the years analyzed. These subpopulations were used as denominators to provide the yearly incidences.

Charlson Comorbidity Index (CCI) was calculated for each patient. This calculation was based on the ICD-10 codes in diagnosis positions from 1 to 20 and applying the algorisms proposed by Sundararajan et al. [17]. We described the prevalence of each single condition included in the CCI and of asthma and pulmonary tuberculosis (See ICD10 codes in Table S1). 
The procedures analyzed included mechanical ventilation (non-invasive and invasive) (ICD-10 codes are shown Table S1).

\subsection{Statistical Analysis}

Incidences were analyzed with Poisson regression models. The measure of association obtained are incidence rate ratios (IRR) along with 95\% confidence intervals (95\% CI).

Descriptive statistical analysis included mean and standard deviation (SD) or median and interquartile range (IQR) for continuous variables, and absolute frequencies and percentages for categorical variables.

Continuous variables were compared using the $t$-test or Mann-Whitney test. Categorical variables were compared using the chi-square test.

We used the Propensity Score Matching (PSM) method to create subpopulations that were comparable based on the distribution of their baseline conditions [18]. We performed three PSM analyses, namely COPD men matched with non-COPD men, COPD women matched with non-COPD women, and COPD men matched with COPD women. The propensity score for each patient, required to do the PSM, was obtained with multivariable logistic regression [18]. In these multivariable models, we included age and all those chronic conditions studied that patients suffered when they were admitted to the hospital. However, due to the important differences in the prevalence of some concomitant conditions between sexes and in order to improve the matching using PSM for COPD men with COPD women, we had to include in the model only those chronic conditions with a prevalence over $5 \%$ in both sexes.

Study subpopulations obtained with PSM were compared with paired $t$-test or $\mathrm{McNe}$ mar's test as required [18].

To assess which of the study variables independently predicted IHM, we conducted multivariable logistic regression. Model construction was done following the steps proposed by Hosmer et al. [19].

The data depuration, statistical analysis, and PSM were done with Stata version 14 software (Stata, College Station, TX, USA).

\subsection{Ethics}

The HDRSNHS is owned by the Spanish Ministry of Health and provided free of charge upon request [20]. Given the characteristics of this registry, which is anonymous, it does not require individual written consent from the patients or ethics committee approval according to the Spanish legislation.

\section{Results}

A total of 500,833 patients aged $\geq 40$ years (59.0\% men and $41.0 \%$ women) were hospitalized in Spain with CAP in the period 2016-2019. COPD was codified in 95,546 patients $(19.1 \%)$. The prevalence of COPD was $26.79 \%$ among men and $7.9 \%$ among women $(p<0.001)$.

\subsection{Incidence of CAP Hospitalizations According to COPD Status}

As can been seen in Table 1, among patients with COPD, we found that the incidence of CAP coding increased significantly from 153.86 in 2016 to 210.71 in 2019 cases per 100,000 persons with COPD $(p<0.001)$. This increment over the study period was also found in patients without COPD $(p<0.001)$. For every year analyzed, the incidence of CAP among COPD subjects was significantly higher than in the population without this condition $(p<0.001)$. After age-and-sex adjusted Poisson regression, the incidence of CAP was 4.42-times higher among the COPD population (IRR 4.42; 95\% CI 4.39-4.45).

The incidence of CAP coding among those with COPD increased from 251.52 cases per 100,000 COPD men population and 51.06 cases per 100,000 COPD women population in 2016 to 353.44 and 74.44 in 2019 , respectively (all $p<0.001$ ). A similar trend was found among women and men without COPD (Table 1). 
Age remained stable over time among those with and without COPD, with slightly higher age among COPD subjects ( $\sim 77$ vs. $\sim 6$ years). The CCI increased significantly over time in both patients with and without COPD (all $p<0.001$ ). LOHS was around 7 days for all years analyzed and in addition to COPD status. IHM remained around 10\% in patients with COPD over time but decreased significantly for patients without COPD from 13.78\% in 2016 to $12.75 \%$ in $2019(p<0.001)$ (Table 1$)$.

Table 1. Trends in the incidence, age distribution, Charlson comorbidity index, and hospital outcomes for patients admitted to Spanish hospitals from the year 2016 to 2019 with community-acquired pneumonia according to presence of chronic obstructive pulmonary disease.

\begin{tabular}{|c|c|c|c|c|c|c|}
\hline & & 2016 & 2017 & 2018 & 2019 & $p$-Value \\
\hline \multirow{2}{*}{$\begin{array}{l}\text { N, Incidence per } \\
100,000 \text { subjects }\end{array}$} & COPD & 21,733 (153.86) & $23,992(169.85)$ & 25,499 (198.68) & $24,322(210.71)$ & $<0.001$ \\
\hline & No COPD & $87,962(36.21)$ & $97,936(40.32)$ & $111,577(44.62)$ & $107,812(41.91)$ & $<0.001$ \\
\hline \multirow{2}{*}{$\begin{array}{l}\text { N, Incidence per } \\
100,000 \text { men }\end{array}$} & COPD & $18,219(251.52)$ & $20,031(276.54)$ & $21,081(327.31)$ & $19,926(353.44)$ & $<0.001$ \\
\hline & No COPD & $47,574(41.19)$ & $52,055(45.07)$ & $59,261(49.7)$ & $57,618(46.84)$ & $<0.001$ \\
\hline \multirow{2}{*}{$\begin{array}{l}\text { N, Incidence per } \\
100,000 \text { women }\end{array}$} & COPD & 3514 (51.06) & 3961 (57.56) & 4418 (69.1) & $4396(74.44)$ & $<0.001$ \\
\hline & No COPD & 40,388 (31.7) & $45,881(36.01)$ & 52,316 (39.99) & $50,194(37.38)$ & $<0.001$ \\
\hline \multirow{2}{*}{ Age, mean (SD) } & COPD & 76.81 (10.69) & 77.31 (10.51) & $76.92(10.79)$ & 76.89 (10.85) & $<0.057$ \\
\hline & No COPD & 75.24 (13.98) & 76.28 (13.61) & 76 (13.76) & $75.91(13.94)$ & $<0.001$ \\
\hline \multirow{2}{*}{$40-64, n(\%)$} & COPD & 2936 (13.51) & 3069 (12.79) & 3574 (14.02) & 3505 (14.41) & $<0.001$ \\
\hline & No COPD & $19,880(22.6)$ & $19,782(20.2)$ & $23,480(21.04)$ & 23,457 (21.76) & $<0.001$ \\
\hline \multirow{2}{*}{$65-74, n(\%)$} & COPD & $5011(23.06)$ & $5434(22.65)$ & $5943(23.31)$ & $5638(23.18)$ & 0.141 \\
\hline & No COPD & $15,232(17.32)$ & $17,137(17.5)$ & $19,746(17.7)$ & 19,037 (17.66) & $<0.001$ \\
\hline \multirow{2}{*}{$75-84, n(\%)$} & COPD & 8168 (37.58) & 8906 (37.12) & 8956 (35.12) & 8355 (34.35) & $<0.001$ \\
\hline & No COPD & $26,164(29.74)$ & $28,540(29.14)$ & $31,524(28.25)$ & $29,041(26.94)$ & $<0.001$ \\
\hline \multirow{2}{*}{$\geq 85, n(\%)$} & COPD & $5618(25.85)$ & $6583(27.44)$ & 7026 (27.55) & $6824(28.06)$ & $<0.001$ \\
\hline & No COPD & $26,686(30.34)$ & 32,477 (33.16) & $36,827(33.01)$ & $36,277(33.65)$ & $<0.001$ \\
\hline \multirow{2}{*}{$\mathrm{CCI}$ index, mean (SD) } & COPD & $1.22(1.1)$ & $1.27(1.1)$ & $1.3(1.13)$ & $1.35(1.15)$ & $<0.001$ \\
\hline & No COPD & $1.14(1.06)$ & $1.21(1.08)$ & $1.21(1.09)$ & $1.25(1.11)$ & $<0.001$ \\
\hline \multirow{2}{*}{ LOHS, median (IQR) } & COPD & $7(7)$ & $7(6)$ & $7(6)$ & $7(6)$ & 0.231 \\
\hline & No COPD & $7(8)$ & $7(7)$ & $7(7)$ & $7(7)$ & 0.076 \\
\hline \multirow{2}{*}{$\mathrm{IHM}, n(\%)$} & COPD & $2279(10.49)$ & $2490(10.38)$ & $2696(10.57)$ & $2412(9.92)$ & 0.081 \\
\hline & No COPD & $12,125(13.78)$ & $13,424(13.71)$ & 14,801 (13.27) & $13,743(12.75)$ & $<0.001$ \\
\hline
\end{tabular}

CAP: community-acquired pneumonia; CCI: Charlson comorbidity index; COPD: chronic obstructive pulmonary disease; LOHS: length of hospital stay; IHM: in-hospital mortality.

\subsection{Clinical Characteristics and Hospital Outcomes for Women and Men with CAP According to} COPD Status

Among women, as can be seen in Table 2, the incidence was significantly higher for those with COPD than in those without COPD (62.49 cases per 100,000 women with COPD vs. 36.30 cases per 100,000 women without COPD, $p<0.001)$, resulting in an adjusted IRR of 1.89 (95\% CI 1.69-2.08).

Before PSM, the mean age was significantly lower among women with COPD (74.77; $\mathrm{SD}=12.65$ years) than non-COPD women $(77.98 ; \mathrm{SD}=13.68$ years $)$, and also the mean CCI was lower. Non-COPD women had higher frequency of dementia (12.28\% vs: $6.22 \%$; $p<0.001$ ), cerebrovascular disease, cancer, and renal disease (Table 2). Women with COPD had a higher prevalence of asthma (10.62\% vs. $9.99 \%: p=0.01)$. 
Table 2. Incidence, age distribution, Charlson comorbidity index, concomitant diseases, and hospital outcomes for women admitted to Spanish hospitals from the year 2016 to 2019 with community-acquired pneumonia according to presence of chronic obstructive pulmonary disease. Results before and after propensity score matching.

\begin{tabular}{|c|c|c|c|c|c|c|}
\hline & \multicolumn{3}{|c|}{ BEFORE PSM } & \multicolumn{3}{|c|}{ AFTER PSM } \\
\hline & COPD & No COPD & $p$-Value & COPD & No COPD & $p$-Value \\
\hline N (incidence per 100,000 women) & $16,289(62.49)$ & $188,779(36.30)$ & $<0.001$ & & & \\
\hline Age, mean (SD) & $74.77(12.65)$ & $77.98(13.68)$ & $<0.001$ & $74.77(12.65)$ & $74.79(12.71)$ & 0.895 \\
\hline $40-64, n(\%)$ & $3914(24.03)$ & $33,942(17.98)$ & $<0.001$ & $3914(24.03)$ & $3919(24.06)$ & 0.948 \\
\hline $50-64, n(\%)$ & $3608(22.15)$ & $26,970(14.29)$ & $<0.001$ & $3608(22.15)$ & $3554(21.82)$ & 0.470 \\
\hline $65-74, n(\%)$ & $4272(26.23)$ & $52,102(27.6)$ & $<0.001$ & $4272(26.23)$ & 4295 (26.37) & 0.772 \\
\hline$\geq 85, n(\%)$ & $4495(27.6)$ & $75,765(40.13)$ & $<0.001$ & $4495(27.6)$ & $4521(27.75)$ & 0.747 \\
\hline CCI index, mean (SD) & $1.06(1.04)$ & $1.14(1.05)$ & $<0.001$ & $1.06(1.04)$ & $1.05(1.03)$ & 0.512 \\
\hline Myocardial infarction, $n(\%)$ & $524(3.22)$ & $5512(2.92)$ & 0.031 & $524(3.22)$ & $506(3.11)$ & 0.569 \\
\hline Congestive heart failure, $n(\%)$ & $4422(27.15)$ & $49,947(26.46)$ & 0.056 & $4422(27.15)$ & $4414(27.1)$ & 0.921 \\
\hline Peripheral vascular disease, $n(\%)$ & $529(3.25)$ & $4606(2.44)$ & $<0.001$ & $529(3.25)$ & $510(3.13)$ & 0.549 \\
\hline Cerebrovascular disease, $n(\%)$ & $687(4.22)$ & $11,334(6)$ & $<0.001$ & $687(4.22)$ & $666(4.09)$ & 0.560 \\
\hline Dementia, $n(\%)$ & $1013(6.22)$ & $23,191(12.28)$ & $<0.001$ & $1013(6.22)$ & $1012(6.21)$ & 0.982 \\
\hline Rheumatoid disease, $n(\%)$ & $552(3.39)$ & $7245(3.84)$ & 0.004 & $552(3.39)$ & $540(3.32)$ & 0.712 \\
\hline Peptic ulcer, $n(\%)$ & $78(0.48)$ & $770(0.41)$ & 0.176 & $78(0.48)$ & $68(0.42)$ & 0.407 \\
\hline Mild liver disease, $n(\%)$ & $869(5.33)$ & $6650(3.52)$ & $<0.001$ & $869(5.33)$ & $859(5.27)$ & 0.805 \\
\hline T2DM, $n(\%)$ & $4288(26.32)$ & $49,060(25.99)$ & 0.348 & $4288(26.32)$ & $4300(26.4)$ & 0.880 \\
\hline Hemiplegia or paraplegia, $n(\%)$ & $82(0.5)$ & $1355(0.72)$ & 0.002 & $82(0.5)$ & $73(0.45)$ & 0.469 \\
\hline Renal disease, $n(\%)$ & $2628(16.13)$ & $34,157(18.09)$ & $<0.001$ & $2628(16.13)$ & $2624(16.11)$ & 0.952 \\
\hline Cancer, $n(\%)$ & $799(4.91)$ & $11,805(6.25)$ & $<0.001$ & $799(4.91)$ & $777(4.77)$ & 0.570 \\
\hline Moderate/severe liver disease, $n(\%)$ & $120(0.74)$ & $1284(0.68)$ & 0.401 & $120(0.74)$ & $108(0.66)$ & 0.425 \\
\hline Metastatic cancer, $n(\%)$ & $371(2.28)$ & $6167(3.27)$ & $<0.001$ & $371(2.28)$ & $367(2.25)$ & 0.882 \\
\hline AIDS, $n(\%)$ & $230(1.41)$ & $1077(0.57)$ & $<0.001$ & $230(1.41)$ & $219(1.34)$ & 0.601 \\
\hline Asthma, $n(\%)$ & $1737(10.62)$ & $19,748(9.99)$ & 0.010 & $1737(10.62)$ & $1632(10.02)$ & 0.105 \\
\hline Pulmonary tuberculosis, $n(\%)$ & $6(0.04)$ & $165(0.08)$ & 0.08 & $6(0.04)$ & $20(0.12)$ & 0.006 \\
\hline $\begin{array}{l}\text { Non-invasive mechanical ventilation, } \\
n(\%)\end{array}$ & $860(5.28)$ & $4689(2.48)$ & $<0.001$ & $860(5.28)$ & $358(2.2)$ & $<0.001$ \\
\hline Invasive mechanical ventilation, $n(\%)$ & $447(2.74)$ & $3620(1.92)$ & $<0.001$ & $447(2.74)$ & $483(2.97)$ & 0.231 \\
\hline LOHS, median (IQR) & $7(6)$ & $7(7)$ & $<0.001$ & $7(6)$ & $7(7)$ & 0.324 \\
\hline $\mathrm{IHM}, n(\%)$ & $1398(8.58)$ & 24,454 (12.95) & $<0.001$ & $1398(8.58)$ & 1811 (11.12) & $<0.001$ \\
\hline
\end{tabular}

PSM: propensity score matching; CAP: community-acquired pneumonia; COPD: chronic obstructive pulmonary disease; CCI: Charlson comorbidity index; T2DM: type 2 diabetes mellitus; AIDS: acquired immune deficiency syndrome; LOHS: length of hospital stay; IHM: in-hospital mortality.

Women with COPD received mechanical ventilation significantly more often than women without COPD (non-invasive: $5.28 \%$ vs. $2.48 \%$; invasive: $2.74 \%$ vs. $1.92 \%$; all $p<0.001)$. The mean LOHS was approximately 7 days in women with and without COPD. The crude IHM was $8.58 \%$ for women with COPD and $12.95 \%$ for non-COPD women $(p<0.001)$.

After PSM, non-invasive mechanical ventilation continued to be more frequent among COPD women; however, invasive ventilation was similar except for COPD status. IHM continued to be lower in non-COPD than in COPD women $(8.58 \%$ vs. $11.12 \%: p<0.001)$.

The results for men appear in Table 3. The crude incidence of CAP was significantly higher in men with COPD than in non-COPD men (298.34 cases per 100,000 men with 
COPD vs. 45.74 cases per 100,000 men without COPD; $p<0.001)$. The adjusted IRR was 5.04 (95\% CI 4.93-5.16).

Table 3. Incidence, age distribution, Charlson comorbidity index, concomitant diseases, and hospital outcomes for men admitted to Spanish hospitals from the year 2016 to 2019 with community-acquired pneumonia according to presence of chronic obstructive pulmonary disease. Results before and after propensity score matching.

\begin{tabular}{|c|c|c|c|c|c|c|}
\hline & \multicolumn{3}{|c|}{ BEFORE PSM } & \multicolumn{3}{|c|}{ AFTER PSM } \\
\hline & COPD & No COPD & $p$-Value & COPD & No COPD & $p$-Value \\
\hline $\mathrm{N}$ (incidence per 100,000 men) & $79,257(298.34)$ & $216,508(45.74)$ & $<0.001$ & & & \\
\hline Age, mean (SD) & $77.44(10.21)$ & $74.05(13.69)$ & $<0.001$ & $77.44(10.21)$ & $77.45(10.21)$ & 0.889 \\
\hline $40-64, n(\%)$ & $9170(11.57)$ & $52,657(24.32)$ & $<0.001$ & $9170(11.57)$ & $9284(11.71)$ & 0.372 \\
\hline $50-64, n(\%)$ & $18,418(23.24)$ & $44,182(20.41)$ & $<0.001$ & $18,418(23.24)$ & $18,351(23.15)$ & 0.690 \\
\hline $65-74, n(\%)$ & $30,113(37.99)$ & $63,167(29.18)$ & $<0.001$ & $30,113(37.99)$ & $29,962(37.80)$ & 0.434 \\
\hline$\geq 85, n(\%)$ & $21,556(27.2)$ & $56,502(26.1)$ & $<0.001$ & $21,556(27.2)$ & $21,660(27.33)$ & 0.344 \\
\hline CCI index, mean (SD) & $1.34(1.13)$ & $1.26(1.11)$ & $<0.001$ & $1.34(1.13)$ & $1.33(1.1)$ & 0.265 \\
\hline Myocardial infarction, $n(\%)$ & $5426(6.85)$ & $13,828(6.39)$ & $<0.001$ & $5426(6.85)$ & $5483(6.92)$ & 0.572 \\
\hline Congestive heart failure, $n(\%)$ & $20,301(25.61)$ & $42,225(19.5)$ & $<0.001$ & $20,301(25.61)$ & $20,278(25.56)$ & 0.894 \\
\hline Peripheral vascular disease, $n(\%)$ & $7453(9.4)$ & $14,950(6.91)$ & $<0.001$ & $7453(9.4)$ & $7336(925)$ & 0.312 \\
\hline Cerebrovascular disease, $n(\%)$ & $4946(6.24)$ & $15,800(7.3)$ & $<0.001$ & $4946(6.24)$ & $5001(6.31)$ & 0.569 \\
\hline Dementia, $n(\%)$ & $4390(5.54)$ & $18,097(8.36)$ & $<0.001$ & $4390(5.54)$ & $4506(5.69)$ & 0.205 \\
\hline Rheumatoid disease, $n(\%)$ & $1484(1.87)$ & $4090(1.89)$ & 0.768 & $1484(1.87)$ & $1606(2.03)$ & 0.027 \\
\hline Peptic ulcer, $n(\%)$ & $635(0.8)$ & $1456(0.67)$ & $<0.001$ & $635(0.8)$ & $586(0.79)$ & 0.159 \\
\hline Mild liver disease, $n(\%)$ & $4903(6.19)$ & $12,851(5.94)$ & 0.011 & $4903(6.19)$ & $4626(5.83)$ & 0.003 \\
\hline T2DM, $n(\%)$ & $26,073(32.9)$ & $60,612(28)$ & $<0.001$ & $26,073(32.9)$ & $25,782(32.53)$ & 0.119 \\
\hline Hemiplegia or paraplegia, $n(\%)$ & $353(0.45)$ & $2232(1.03)$ & $<0.001$ & $353(0.45)$ & $407(0.51)$ & 0.049 \\
\hline Renal disease, $n(\%)$ & $17,108(21.59)$ & $41,701(19.26)$ & $<0.001$ & $17,108(21.59)$ & $16,882(21.35)$ & 0.167 \\
\hline Cancer, $n(\%)$ & $7776(9.81)$ & $24,040(11.1)$ & $<0.001$ & $7776(9.81)$ & $7921(9.99)$ & 0.222 \\
\hline $\begin{array}{l}\text { Moderate/severe liver disease, } \\
n(\%)\end{array}$ & $796(1)$ & $3088(1.43)$ & $<0.001$ & $796(1)$ & $699(0.88)$ & 0.011 \\
\hline Metastatic cancer, $n(\%)$ & $3499(4.41)$ & $13,912(6.43)$ & $<0.001$ & $3499(4.41)$ & $3611(4.55)$ & 0.174 \\
\hline AIDS, $n(\%)$ & $632(0.8)$ & $2739(1.27)$ & $<0.001$ & $632(0.8)$ & $665(0.81)$ & 0.933 \\
\hline Asthma, $n(\%)$ & $2041(3.04)$ & $6565(3.03)$ & 0.675 & $2041(3.04)$ & $1932(2.44)$ & 0.079 \\
\hline Pulmonary tuberculosis, $n(\%)$ & $83(0.10)$ & $326(0.15)$ & 0.003 & $83(0.10)$ & $94(0.12)$ & 0.408 \\
\hline $\begin{array}{l}\text { Non-invasive mechanical } \\
\text { ventilation, } n(\%)\end{array}$ & $3365(4.25)$ & $5444(2.51)$ & $<0.001$ & $3365(4.25)$ & $1806(2.41)$ & $<0.001$ \\
\hline $\begin{array}{l}\text { Invasive mechanical ventilation, } \\
n(\%)\end{array}$ & $1813(2.29)$ & $6778(3.13)$ & $<0.001$ & $1813(2.29)$ & $2053(2.59)$ & $<0.001$ \\
\hline LOHS, median (IQR) & $7(6)$ & $7(8)$ & 0.063 & $7(6)$ & $7(8)$ & 0.0102 \\
\hline $\mathrm{IHM}, n(\%)$ & 8479 (10.7) & 29639 (13.69) & $<0.001$ & $8479(10.70)$ & $11,424(14.41)$ & $<0.001$ \\
\hline
\end{tabular}

PSM: propensity score matching; CAP: community-acquired pneumonia; COPD: chronic obstructive pulmonary disease; CCI: Charlson comorbidity index; T2DM: type 2 diabetes mellitus; AIDS: acquired immune deficiency syndrome; LOHS: length of hospital stay; IHM: in-hospital mortality.

Before PSM, significant differences in the distribution age, and comorbidities between men with and without COPD were found. Men with COPD were older (77.44 years vs. 74.05 years) and had higher mean CCI (1.34 vs. 1.26) and higher prevalence of myocardial infarction, T2DM, congestive heart failure, renal disease, peripheral vascular disease, peptic ulcer, and mild liver disease. However, those without COPD had a higher prevalence 
of dementia, cerebrovascular disease, and cancer. Men with COPD received more noninvasive mechanical ventilation $(4.25 \%$ vs. $2.51 \% ; p<0.001)$ but received less invasive mechanical ventilation $(2.29 \%$ vs. $3.13 \% ; p<0.001)$. LOHS was around 7 days in both men with and without COPD. Non-COPD men had higher crude IHM than men with COPD (13.69\% vs. $10.7 \%, p<0.001)$.

After PSM, we found that among COPD men, non-invasive mechanical ventilation continued to be more frequent, and invasive ventilation continued to be less frequent. After PSM, the IHM continued to be higher in non-COPD men $(14.41 \%$ vs. $10.70 \% ; p<0.001)$.

\subsection{Sex Differences for COPD Patients Hospitalized with CAP}

As can be seen in Table 4, the incidence was significantly lower in women than in men with COPD. The overall age-adjusted incidence of CAP over the period 2016-2019 was 4.77 times higher among men with COPD than among women with COPD (IRR 4.77; 95\% CI 4.69-4.85 according to the Poisson regression analysis). The IRR among those without COPD was 1.26 (95\% 1.25-1.26).

Table 4. Incidence, age distribution, Charlson comorbidity index, concomitant diseases and hospital outcomes for men and women suffering chronic obstructive pulmonary disease admitted to Spanish hospitals from year 2016 to 2019 with community-acquired pneumonia. Results before and after propensity score matching.

\begin{tabular}{|c|c|c|c|c|c|c|}
\hline & \multicolumn{3}{|c|}{ BEFORE PSM } & \multicolumn{3}{|c|}{ AFTER PSM } \\
\hline & COPD MEN & $\begin{array}{c}\text { COPD } \\
\text { WOMEN }\end{array}$ & $p$-Value & COPD MEN & $\begin{array}{c}\text { COPD } \\
\text { WOMEN }\end{array}$ & $p$-Value \\
\hline $\mathrm{N}$ (incidence per 100,000 subjects) & $79,257(298.34)$ & $16,289(62.49)$ & & & & \\
\hline Age, mean (SD) & $77.44(10.21)$ & $74.77(12.65)$ & $<0.001$ & $74.59(11.95)$ & $74.77(12.64)$ & $<0.062$ \\
\hline $40-64, n(\%)$ & $9170(11.57)$ & $3914(24.03)$ & $<0.0010$ & $3889(23.88)$ & $3910(24.01)$ & 0.745 \\
\hline $50-64, n(\%)$ & $18,418(23.24)$ & $3608(22.15)$ & 0.002 & $3585(22.04)$ & $3608(22.16)$ & 0.759 \\
\hline $65-74, n(\%)$ & $30,113(37.99)$ & $4272(26.23)$ & $<0.001$ & $4320(26.53)$ & $4272(26.23)$ & 0.546 \\
\hline$\geq 85, n(\%)$ & $21,556(27.2)$ & 4495 (27.6) & 0.299 & $4491(27.58)$ & $4495(27.60)$ & 0.960 \\
\hline CCI index, mean (SD) & $1.34(1.13)$ & $1.06(1.04)$ & $<0.001$ & $1.07(1.06)$ & $1.06(1.04)$ & 0.120 \\
\hline Myocardial infarction, $n(\%)$ & $5426(6.85)$ & $524(3.22)$ & $<0.001$ & $601(3.69)$ & $524(3.22)$ & 0.019 \\
\hline Congestive heart failure, $n(\%)$ & $20,301(25.61)$ & $4422(27.15)$ & $<0.001$ & $4400(27.02)$ & $4422(27.15)$ & 0.783 \\
\hline Peripheral vascular disease, $n(\%)$ & $7453(9.4)$ & $529(3.25)$ & $<0.001$ & $723(4.44)$ & $529(3.25)$ & $<0.001$ \\
\hline Cerebrovascular disease, $n(\%)$ & $4946(6.24)$ & $687(4.22)$ & $<0.001$ & $708(4.35)$ & $687(4.22)$ & 0565 \\
\hline Dementia, $n(\%)$ & $4390(5.54)$ & $1013(6.22)$ & 0.001 & $956(5)$ & $1013(6.22)$ & 0.185 \\
\hline Rheumatoid disease, $n(\%)$ & $1484(1.87)$ & $552(3.39)$ & $<0.001$ & $458(2.81)$ & $552(3.39)$ & 0.002 \\
\hline Peptic ulcer, $n(\%)$ & $635(0.8)$ & $78(0.48)$ & $<0.001$ & $97(0.60)$ & $78(0.48)$ & 0.150 \\
\hline Mild liver disease, $n(\%)$ & $4903(6.19)$ & $869(5.33)$ & $<0.001$ & $932(5.72)$ & $868(5.33)$ & 0.127 \\
\hline T2DM, $n(\%)$ & $26,073(32.9)$ & $4288(26.32)$ & $<0.001$ & $4317(26.50)$ & $4288(26.33)$ & 0.716 \\
\hline Hemiplegia or paraplegia, $n(\%)$ & $353(0.45)$ & $82(0.5)$ & 0.316 & $60(0.37)$ & $82(0.5)$ & 0.064 \\
\hline Renal disease, $n(\%)$ & $17,108(21.59)$ & $2628(16.13)$ & $<0.001$ & $2673(16.41)$ & $2624(16.11)$ & 0.498 \\
\hline Cancer, $n(\%)$ & $7776(9.81)$ & $799(4.91)$ & $<0.001$ & $875(5.373)$ & $799(4.91)$ & 0.056 \\
\hline $\begin{array}{l}\text { Moderate/severe liver disease, } \\
n(\%)\end{array}$ & $796(1)$ & $120(0.74)$ & 0.001 & $156(0.96)$ & $120(0.74)$ & 0.030 \\
\hline Metastatic cancer, $n(\%)$ & $3499(4.41)$ & $371(2.28)$ & $<0.001$ & $446(2.74)$ & $371(2.28)$ & 0.008 \\
\hline AIDS, $n(\%)$ & $632(0.8)$ & $230(1.41)$ & $<0.001$ & $180(1,11)$ & $229(1.41)$ & 0.013 \\
\hline Asthma, $n(\%)$ & $2041(3.04)$ & $1737(10.62)$ & $<0.001$ & $1635(10.04)$ & $1737(10.62)$ & 0.065 \\
\hline
\end{tabular}


Table 4. Cont.

\begin{tabular}{|c|c|c|c|c|c|c|}
\hline & \multicolumn{3}{|c|}{ BEFORE PSM } & \multicolumn{3}{|c|}{ AFTER PSM } \\
\hline & COPD MEN & $\begin{array}{l}\text { COPD } \\
\text { WOMEN }\end{array}$ & $p$-Value & COPD MEN & $\begin{array}{l}\text { COPD } \\
\text { WOMEN }\end{array}$ & $p$-Value \\
\hline Pulmonary tuberculosis, $n(\%)$ & $83(0.10)$ & $6(0.04)$ & 0.013 & $16(0.10)$ & $6(0.04)$ & 0.050 \\
\hline $\begin{array}{l}\text { Non-invasive mechanical } \\
\text { ventilation, } n(\%)\end{array}$ & $3365(4.25)$ & $860(5.28)$ & $<0.001$ & $711(4.36)$ & $860(5.28)$ & $<0.001$ \\
\hline $\begin{array}{l}\text { Invasive mechanical ventilation, } \\
n(\%)\end{array}$ & $1813(2.29)$ & $447(2.74)$ & $<0.001$ & $450(2.76)$ & $447(2.74)$ & 0.919 \\
\hline LOHS, median (IQR) & $7(6)$ & $7(6)$ & 0.044 & $7(6)$ & $7(6)$ & 0.859 \\
\hline $\mathrm{IHM}, n(\%)$ & $8479(10.7)$ & $1398(8.58)$ & $<0.001$ & $1566(9.61)$ & $1398(8.58)$ & 0.001 \\
\hline
\end{tabular}

PSM: propensity score matching; CAP: community-acquired pneumonia; COPD: chronic obstructive pulmonary disease; CCI: Charlson comorbidity index; T2DM: type 2 diabetes mellitus; AIDS: acquired immune deficiency syndrome; LOHS: length of hospital stay; IHM: in-hospital mortality.

Men with COPD were older $(77.44 \pm 10.21$ years vs. $74.77 \pm 12.65$ years; $p<0.001)$ and with a higher mean CCI $(1.34 \pm 1.13$ vs. $1.06 \pm 1.04)$ than COPD women. Men more frequently had a code for myocardial infarction, T2DM, cerebrovascular disease, peripheral vascular disease, liver disease (mild and moderate/severe), renal disease, cancer, and metastatic cancer.

However, congestive heart failure, dementia, rheumatoid disease, peptic ulcer, and AIDS were more prevalent in women than in men. The prevalence of asthma was over three times higher among women with COPD than men with COPD $(10.62 \%$ vs. 3.04 ; $p<0.001$ ).

The difference in IHM was statistically significant $(p<0.001)$ before and after PSM, with proportions of $9.61 \%$ for men with COPD and $8.58 \%$ for women with COPD after PSM.

3.4. Variables Associated with Dying during Hospitalization among Women and Men with CAP According to COPD Status

As can been seen in Table 5, the IHM increased with age and with the suffering of congestive heart failure, renal disease, cancer or metastatic cancer, cerebrovascular disease, moderate or severe liver disease, hemiplegia or paraplegia, and dementia, among men and women with COPD. Myocardial infarction was only a factor associated with IHM in men, but not in women, with COPD (OR 1.18; 95\% CI 1.08-1.28).

Table 5. Multivariable analysis to assess time trend and to identify study variables independently associated with in-hospital mortality among men and women hospitalized with CAP in Spain from 2016 to 2019.

\begin{tabular}{lccc}
\hline & MEN & WOMEN & BOTH \\
\hline 40-64 years old, $n(\%)$ & OR (95\% CI) & OR (95\% CI) & OR (95\% CI) \\
\hline 50-64 years old, $n(\%)$ & 1 & 1 & 1 \\
\hline 65-74 years old, $n(\%)$ & $1.40(1.26-1.56)$ & $1.89(1.49-2.39)$ & $1.51(1.38-1.67)$ \\
\hline$\geq 85$ years old, $n(\%)$ & $2.14(1.94-2.36)$ & $3.39(2.71-4.25)$ & $2.35(2.15-2.58)$ \\
\hline Myocardial infarction & $3.54(3.2-3.93)$ & $6.91(5.52-8.64)$ & $4.03(3.67-4.42)$ \\
\hline Congestive heart failure & $1.18(1.08-1.28)$ & NS & $1.17(1.07-1.27)$ \\
\hline Cerebrovascular disease & $1.42(1.35-1.49)$ & $1.49(1.32-1.69)$ & $1.44(1.37-1.51)$ \\
\hline Dementia & $1.54(1.41-1.67)$ & $1.63(1.3-2.05)$ & $1.55(1.43-1.67)$ \\
\hline T2DM & $1.99(1.83-2.16)$ & $1.92(1.61-2.3)$ & $1.99(1.84-2.14)$ \\
\hline
\end{tabular}


Table 5. Cont.

\begin{tabular}{lccc}
\hline & MEN & WOMEN & BOTH \\
\hline Hemiplegia or paraplegia & OR (95\% CI) & OR (95\% CI) & OR (95\% CI) \\
\hline Renal disease & $2.71(2.09-3.52)$ & $3.55(2.04-6.2)$ & $2.83(2.24-3.59)$ \\
\hline Cancer & $1.23(1.17-1.3)$ & $1.19(1.03-1.38)$ & $1.23(1.17-1.29)$ \\
\hline Moderate/severe liver disease & $2.07(1.93-2.22)$ & $2.23(1.77-2.81)$ & $2.08(1.95-2.22)$ \\
\hline Metastatic cancer & $2.93(2.43-3.52)$ & $2.4(1.42-4.06)$ & $2.9(2.44-3.45)$ \\
\hline Asthma & $5.32(4.89-5.8)$ & $8.95(6.86-11.68)$ & $5.6(5.16-6.07)$ \\
\hline Non-invasive mechanical ventilation & $2.52(2.3-2.77)$ & $2.85(2.33-3.49)$ & $2.57(2.36-2.79)$ \\
\hline Invasive mechanical ventilation & $8.65(7.78-9.61)$ & $10.35(8.12-13.2)$ & $8.82(8.01-9.72)$ \\
\hline 2016 & 1 & 1 & 1 \\
\hline 2017 & $0.98(0.91-1.04)$ & $0.89(0.76-1.05)$ & $0.96(0.91-1.03)$ \\
\hline 2018 & $0.99(0.93-1.06)$ & $0.84(0.72-0.99)$ & $0.97(0.91-1.03)$ \\
\hline 2019 & $0.91(0.85-0.98)$ & $0.71(0.6-0.84)$ & $0.88(0.82-0.94)$ \\
\hline Women & NA & NA & 1 \\
\hline Men & NA & NA & $1.12(1.05-1.19)$ \\
\hline
\end{tabular}

T2DM: type 2 diabetes mellitus; NA: not applicable; NS: not significant. The absence of the condition or procedure is used as the reference category.

In both men and women with COPD, the presence of T2DM reduced the IHM (OR $0.85 \% ; 95 \%$ CI $0.81-0.89$ and OR $0.83 ; 95 \%$ CI 0.73-0.95, respectively). Among women with COPD, the presence of asthma reduced the risk of dying in the hospital (OR 0.74; 95\% CI $0.62-0.89)$.

The need for mechanical ventilation (non-invasive and invasive) during admission increased the risk of IHM in COPD patients irrespective of sex (OR 2.56; 95\% CI 2.36-2.79 and OR 8.71; 95\% CI 7.9-9.6, respectively).

Over time, the IHM decreased significantly in both men and women with COPD. Finally, as found with the PSM, men with COPD were significantly more likely to die in hospital than were COPD women (OR 1.13; 95\% CI 1.07-1.21).

In Table S2 are shown the variables associated with IHM among the non-COPD population according to sex. The variables that increased and reduced the risk of dying were the same described for the men and women with COPD. However, among non-COPD men, the risk of dying increased among those suffering from AIDS and decreased if a code for asthma was recorded. These two significant associations were not found among men with COPD

As reported for those with COPD, the IHM was higher among men than women, and the IHM decreased significantly from 2016 to 2019 for both sexes.

\section{Discussion}

In this nationwide population-based cohort study, we found that the incidence of CAP increased significantly from 2016 to 2019, both in COPD and non-COPD patients. It is important to note that ICD-10 was implemented in Spain as a reference classification for clinical coding from 1 January 2016, and it is possible that coding may became more accurate over time. However, these results are in agreement with studies carried out in periods priors to ours, and they did not appear to be fully explained by demographic changes or by an increase in co-existing conditions [21-23]. In addition, we reported that after multivariable adjustment in the population with COPD, the incidence of CAP was four times higher than in the population without COPD. In previous studies, prior COPD exacerbation, COPD severity, low body mass index, more comorbid conditions, use of 
inhaled corticosteroid (ICS), smoking habit, suboptimal immunization, and higher age have been identified as risk factors for CAP in persons suffering from COPD [4,13,24].

By sex, the incidence of CAP increased in both men and women with and without COPD, although it was also significantly higher in men and women with COPD than in those without COPD, especially among men. Other studies have also shown that the incidence of CAP is higher in men than in women $[3,25]$, but there are no previous studies in the literature that assess the influence of sex on the relationship between CAP and COPD. In any case, it is possible that biological, cultural, and behavioral factors, as well as socioeconomic differences, could partly justify these results in patients with CAP and COPD [12].

We agree with Cavallazzi et al., finding that among CAP, those with concomitant COPD suffer more comorbidities and are older [26]. In this regard, we observed that the mean age and the mean CCI increased significantly over time in addition to COPD status. Among men, we did not detect significant differences in mean age between those with and without COPD, but comorbidity was slightly higher in COPD patients. Among women, we did not detect significant differences after matching in age or comorbidity between those with and without COPD. Furthermore, we observed that men with CAP and COPD had a higher mean CCI than women with both conditions. In the same way, Alsawas et al. [27] analyzed the sex disparities between hospitalized patients with pneumonia and found that the prevalence of comorbidities was significantly different between men and women, such that men had multi-comorbidities more frequently than women. In the same study, multi-comorbidities had a significant effect on readmission at 30 days in men, but not in women.

Despite the increase in comorbidity, IHM decreased significantly from year 2016 to year 2019 in COPD in women and men, suggesting an improvement in the clinical management of COPD over time. A remarkable result is that the IHM was lower in women and men who suffered COPD hospitalized with CAP than in those without this chronic disease.

In the studies published to date, it is still unclear whether COPD is associated with a better or worse survival after CAP [9-11]. The opposite conclusions found in these studies may be due to differences in the study design, sample population characteristics regarding age and sex, prevalence of smoking habit, COPD severity, associated comorbidities, established treatment, and use of healthcare resources [28]. However, the lower mortality among COPD patients has been reported by Dusemund et al., who conducted an age-sex matched case-control study in Switzerland from 2002 to 2010 analyzing 17.075 patients [29]. Furthermore, two national population-based cohort studies conducted in Germany and Spain reported COPD as a protective factor for mortality after CAP [9,23]. The lowerthan-expected mortality among COPD patients could be due to multiple reasons including biological factors such as a different immune response or a beneficial anti-inflammatory effect caused by inhaled corticosteroids [26,29]. Other explanation could be the increased awareness of patients with COPD and clinicians treating patients with this condition that would result in an earlier diagnosis, treatment, and hospital admission with a less severe CAP. Despite the lack of a damaging short-term effect of COPD, it is possible that COPD patients have a worse mid and long-term mortality after CAP, as recently described by Bordon et al. [24]. As reported by other authors [11,30,31], after multivariable adjustment, the short-term mortality did not differ among COPD patients. Notwithstanding these findings, some studies detected an association between COPD and mortality in patients with CAP $[8,10,32]$, and in others, this association became not significant when other risk factors were included in the model [33].

In the future large prospective studies should be done to clarify the role of COPD on the outcome of CAP [2].

On the other hand, we found that men with COPD were significantly more likely to die in hospital than were COPD women. Several authors have also shown that CAP is more severe and results in higher mortality among men than women, especially in older 
age [12,34]. Sex-differences might be due to biologic differences [12] but also to many other factors, including access to care [35].

To our knowledge, few studies have assessed risk factors of mortality after CAP in patients hospitalized with concomitant COPD [36]. We identified that the risk factors for mortality among men and women with COPD were older age, suffering congestive heart failure, renal disease, cancer or metastatic cancer, cerebrovascular disease, moderate or severe liver disease, hemiplegia or paraplegia, and dementia. However, the presence of T2DM reduced the IHM in both groups. T2DM is strongly associated with high body mass index, and the existence of an obesity paradox has been suggested for CAP [37]. The obesity paradox refers to epidemiological evidence showing that obesity in patients may have a protective effect that leads to decreased mortality among hospitalized patients [37].

Among women with COPD, the presence of concomitant asthma was associated with lower IHM after CAP. In a previous study, Yamauchi et al. also demonstrated that in-hospital mortality was higher in patients with COPD alone than in those with ACOS [38]. Both asthma and COPD patients are ICS users and, as previously described, association between asthma/COPD and lower IHM may be due to selection bias [39]. However, the association persisted even after taking into account known covariates by applying multivariate regression analysis. Another possible explanation for the protective effect of asthma on the IHM may be that patients with this concomitant condition are admitted with less severe CAP due to the asthmatic symptomatology, but this is unclear.

As in other studies [40], the need for mechanical ventilation during admission also increased the risk of IHM in COPD-CAP patients irrespective of sex.

Our study has strengths and limitations. The strengths include the population-based design and the large sample size, covering the entire population of Spain. The main limitation is related to use of an administrative database. Therefore, we lacked information on clinical variables, such as severity of pneumonia, degree of airway obstruction, antibiotic therapy, use of corticosteroids, and history of influenza or pneumococcal immunizations. In addition, even if our database had high specificity, is possible that some conditions and procedures were under-reported [41]. Furthermore, in our investigation we could not analyze the asthma-COPD overlap syndrome (ACOS) because, unfortunately, there is no specific code for ACOS in the ICD10 [42].

Finally, we did not evaluate the influence of COPD in CAP patients that did not require hospitalization.

\section{Conclusions}

In conclusion, we found that COPD increased the incidence of CAP, although its effect was higher in men than in women. IHM was lower in COPD patients of both sexes than in those without COPD. Men with COPD were significantly more likely to die in hospital than COPD women. Our results indicate that sex may be an important determinant of incidence and mortality of pneumonia in COPD patients, adding information to the existing literature in this field.

Supplementary Materials: The following is available online at https:/ / www.mdpi.com/article/10.3 390/jcm10214889/s1, Table S1: ICD-10 codes for diagnosis and therapeutic procedures. Table S2: Multivariable analysis of factors associated with in-hospital mortality during admissions for communityacquired pneumonia, among patients not suffering chronic obstructive pulmonary disease according to sex. Figure S1: Flow chart of patient selection and hospital outcome according COPD status and sex.

Author Contributions: Conceptualization, J.d.M.-D., A.L.-d.-A. and R.J.-G.; Data curation, V.H.-B., D.C.-A. and N.P.-F.; Formal analysis, V.H.-B., D.C.-A. and J.M.d.M.-Y.; Methodology, M.L.-H. and J.W.; Writing-original draft preparation, J.d.M.-D., A.L.-d.-A. and R.J.-G.; Writing-review and editing, J.M.d.M.-Y., N.P.-F., M.L.-H. and J.W. All authors have read and agreed to the published version of the manuscript.

Funding: This research received no external funding. 
Institutional Review Board Statement: Not applicable.

Informed Consent Statement: Not applicable.

Data Availability Statement: According to the contract signed with the Spanish Ministry of Health and Social Services, which provided access to the databases from the Hospital Discharge Records of the Spanish National Health System (In Spanish: Conjunto Mínimo Basico de Datos (CMBD)), we cannot share the databases with any other investigator, and we have to destroy the databases once the investigation has concluded. Consequently, we cannot upload the databases to any public repository. However, any investigator can apply for access to the databases by filling out the questionnaire available at http:/ / www.msssi.gob.es/estadEstudios/estadisticas/estadisticas/estMinisterio/ SolicitudCMBDdocs/Formulario_Peticion_Datos_CMBD.pdf (accessed on 16 March 2021). All other relevant data are included in the paper.

Conflicts of Interest: The authors declare no conflict of interest.

\section{References}

1. Welte, T.; Torres, A.; Nathwani, D. Clinical and economic burden of community-acquired pneumonia among adults in Europe. Thorax 2012, 67, 71-79. [CrossRef]

2. Liu, D.S.; Han, X.D.; Liu, X.D. Current status of community-acquired pneumonia in patients with chronic obstructive pulmonary disease. Chin. Med. J. 2018, 131, 1086-1091. [CrossRef]

3. Millett, E.R.C.; Quint, J.K.; Smeeth, L.; Daniel, R.M.; Thomas, S.L. Incidence of community-acquired lower respiratory tract infections and pneumonia among older adults in the United Kingdom: A population-based study. PLoS ONE 2013, 8, e75131. [CrossRef]

4. Müllerova, H.; Chigbo, C.; Hagan, G.W.; Woodhead, M.A.; Miravitlles, M.; Davis, K.J.; Wedzicha, J.A. The natural history of community-acquired pneumonia in COPD patients: A population database analysis. Respir. Med. 2012, 106, 1124-1133. [CrossRef]

5. Lozano, R.; Naghavi, M.; Foreman, K.; Lim, S.; Shibuya, K.; Aboyans, V.; Abraham, J.; Adair, T.; Aggarwal, R.; Ahn, S.Y.; et al. Global and regional mortality from 235 causes of death for 20 age groups in 1990 and 2010: A systematic analysis for the Global Burden of Disease Study 2010. Lancet 2012, 380, 2095-2128. [CrossRef]

6. Søgaard, M.; Madsen, M.; Løkke, A.; Hilberg, O.; Sørensen, H.T.; Thomsen, R.W. Incidence and outcomes of patients hospitalized with COPD exacerbation with and without pneumonia-. Int. J. Chron. Obstruct. Pulmon. Dis. 2016, 11, 455-465. [CrossRef]

7. Crim, C.; Calverley, P.M.; Anderson, J.A.; Celli, B.; Ferguson, G.T.; Jenkins, C.; Jones, P.W.; Willits, L.R.; Yates, J.C.; Vestbo, J. Pneumonia risk in COPD patients receiving inhaled corticosteroids alone or in combination: TORCH study results. Eur. Respir. J. 2009, 34, 641-647. [CrossRef]

8. Molinos, L.; Clemente, M.G.; Miranda, B.; Alvarez, C.; del Busto, B.; Cocina, B.R.; Alvarez, F.; Gorostidi, J.; Orejas, C.; ASTURPAR Group. Community-acquired pneumonia in patients with and without chronic obstructive pulmonary disease. J. Infect. 2009, 58, 417-424. [CrossRef]

9. $\quad$ Ewig, S.; Birkner, N.; Strauss, R.; Schaefer, E.; Pauletzki, J.; Bischoff, H.; Schraeder, P.; Welte, T.; Hoeffken, G. New perspectives on community-acquired pneumonia in 388406 patients. Results from a nationwide mandatory performance measurement programme in healthcare quality. Thorax 2009, 64, 1062-1069. [CrossRef]

10. Restrepo, M.I.; Mortensen, E.M.; Pugh, J.A.; Anzueto, A. COPD is associated with increased mortality in patients with communityacquired pneumonia. Eur. Respir. J. 2006, 28, 346-351. [CrossRef]

11. Snijders, D.; van der Eerden, M.; de Graaff, C.; Boersma, W. The influence of COPD on mortality and severity scoring in community-acquired pneumonia. Respiration 2010, 79, 46-53. [CrossRef]

12. Barbagelata, E.; Cillóniz, C.; Dominedò, C.; Torres, A.; Nicolini, A.; Solidoro, P. Gender differences in community-acquired pneumonia. Minerva. Med. 2020, 111, 153-165. [CrossRef]

13. Williams, N.P.; Coombs, N.A.; Johnson, M.J.; Josephs, L.K.; Rigge, L.A.; Staples, K.J.; Thomas, M.; Wilkinson, T.M. Seasonality, risk factors and burden of community-acquired pneumonia in COPD patients: A population database study using linked health care records. Int. J. Chron. Obstruct. Pulmon. Dis. 2017, 12, 313-322. [CrossRef]

14. Ministerio de Sanidad, Servicios Sociales e Igualdad. Real Decreto 69/2015, de 6 de Febrero, por el que se Regula el Registro de Actividad de Atención Sanitaria Especializada. (Spanish National Hospital Discharge Database); Boletin Oficial del Estado, 2015; Volume 35, pp. 10789-10809. Available online: https://www.mscbs.gob.es/estadEstudios/estadisticas/docs/BOE_RD_69_2015_ RAE_CMBD.pdf (accessed on 12 May 2021).

15. Instituto Nacional de Estadistica. Population Estimations. Available online: https://www.ine.es/jaxiT3/Tabla.htm?t=. (accessed on 16 March 2021).

16. Instituto Nacional de Estadistica. National Health Survey 2017, SNHS 2017 Methodology. 2017. Available online: https: / / www.ine.es/en/metodologia/t15/t153041917_en.pdf (accessed on 16 March 2021).

17. Sundararajan, V.; Henderson, T.; Perry, C.; Muggivan, A.; Quan, H.; Ghali, W.A. New ICD-10 version of the Charlson comorbidity index predicted in-hospital mortality. J. Clin. Epidemiol. 2004, 57, 1288-1294. [CrossRef] 
18. Austin, P.C. Comparing paired vs non-paired statistical methods of analyses when making inferences about absolute risk reductions in propensity-score matched samples. Stat. Med. 2011, 30, 1292-1301. [CrossRef]

19. Hosmer, D.W.; Lemeshow, S.; Sturdivant, R.X. Applied Logistic Regression, 3rd ed.; John Wiley \& Sons, Inc.: Hoboken, NJ, USA, 2013.

20. De Sanidad, M.; Consumo y Bienestar Social. Solicitud de Extracción de Datos-Extraction Request (Spanish National Hospital Discharge Database). Available online: https://www.mscbs.gob.es/estadEstudios/estadisticas/estadisticas/estMinisterio/ SolicitudCMBDdocs/2018_Formulario_Peticion_Datos_RAE_CMBD.pdf (accessed on 12 May 2021).

21. Trotter, C.L.; Stuart, J.M.; George, R.; Miller, E. Increasing hospital admissions for pneumonia, England. Emerg. Infect. Dis. 2008, 14, 727-733. [CrossRef]

22. Quan, T.P.; Fawcett, N.J.; Wrightson, J.M.; Finney, J.; Wyllie, D.; Jeffery, K.; Jones, N.; Shine, B.; Clarke, L.; Crook, D.; et al. Infections in Oxfordshire Research Database (IORD). Increasing burden of community-acquired pneumonia leading to hospitalisation, 1998-2014. Thorax 2016, 71, 535-542. [CrossRef]

23. De Miguel-Díez, J.; López-de-Andrés, A.; Hernández-Barrera, V.; Jiménez-Trujillo, I.; Méndez-Bailón, M.; de Miguel-Yanes, J.M.; Jiménez-García, R. Impact of COPD on outcomes in hospitalized patients with community-acquired pneumonia: Analysis of the Spanish national hospital discharge database (2004-2013). Eur. J. Intern. Med. 2017, 43, 69-76. [CrossRef]

24. Bordon, J.; Slomka, M.; Gupta, R.; Furmanek, S.; Cavallazzi, R.; Sethi, S.; Niederman, M.; Ramirez, J.A.; University of Louisville Pneumonia Study Group. Hospitalization due to community-acquired pneumonia in patients with chronic obstructive pulmonary disease: Incidence, epidemiology and outcomes. Clin. Microbiol. Infect. 2020, 26, 220-226. [CrossRef]

25. López-de-Andrés, A.; Albaladejo-Vicente, R.; de Miguel-Diez, J.; Hernández-Barrera, V.; Ji, Z.; Zamorano-León, J.J.; LopezHerranz, M.; Carabantes Alarcon, D.; Jimenez-Garcia, R. Gender differences in incidence and in-hospital outcomes of communityacquired, ventilator-associated and nonventilator hospital-acquired pneumonia in Spain. Int. J. Clin. Pract. 2021, 75, e13762. [CrossRef]

26. Cavallazzi, R.; Ramirez, J. Community-acquired pneumonia in chronic obstructive pulmonary disease. Curr. Opin. Infect. Dis. 2020, 33, 173-181. [CrossRef]

27. Alsawas, M.; Wang, Z.; Murad, M.H.; Yousufuddin, M. Gender disparities among hospitalised patients with acute myocardial infarction, acute decompensated heart failure or pneumonia: Retrospective cohort study. BMJ Open 2019, 9, e022782. [CrossRef]

28. Bonnesen, B.; Baunbæk Egelund, G.; Vestergaard Jensen, A.; Andersen, S.; Trier Petersen, P.; Rohde, G.; Ravn, P. Is chronic obstructive pulmonary disease a risk factor for death in patients with community acquired pneumonia? Infect. Dis. 2019, 51, 340-347. [CrossRef]

29. Dusemund, F.; Chronis, J.; Baty, F.; Christian Albrich, W.; Hugo Brutsche, M. The outcome of community-acquired pneumonia in patients with chronic lung disease: A case-control study. Swiss Med. Wkly. 2014, 144, w14013. [CrossRef]

30. Liapikou, A.; Polverino, E.; Ewig, S.; Cillóniz, C.; Marcos, M.A.; Mensa, J.; Bello, S.; Martin-Loeches, I.; Menéndez, R.; Torres, A. Severity and outcomes of hospitalised community-acquired pneumonia in COPD patients. Eur. Respir. J. 2012, 39, 855-861. [CrossRef]

31. Kew, K.M.; Seniukovich, A. Inhaled steroids and risk of pneumonia for chronic obstructive pulmonary disease. Cochrane Database Syst. Rev. 2014, 3, CD010115. [CrossRef]

32. Neupane, B.; Walter, S.D.; Krueger, P.; Marrie, T.; Loeb, M. Predictors of inhospital mortality and re-hospitalization in older adults with community-acquired pneumonia: A prospective cohort study. BMC Geriatr. 2010, 10, 22. [CrossRef]

33. Braeken, D.C.W.; Franssen, F.M.E.; Schütte, H.; Pletz, M.W.; Bals, R.; Martus, P.; Rohde, G.G.U.; on behalf of the CAPNETZ Study Group. Increased severity and mortality of CAP in COPD: Results from the German Competence Network, CAPNETZ. Chronic. Obstr. Pulm. Dis. 2015, 2, 131-140. [CrossRef]

34. Kaplan, V.; Angus, D.C.; Griffin, M.F.; Clermont, G.; Scott Watson, R.; Linde-Zwirble, W.T. Hospitalized community-acquired pneumonia in the elderly: Age- and sex-related patterns of care and outcome in the United States. Am. J. Respir. Crit. Care Med. 2002, 165, 766-772. [CrossRef]

35. Giles, W.H.; Anda, R.F.; Casper, M.L.; Escobedo, L.G.; Taylor, H.A. Race and sex differences in rates of invasive cardiac procedures in US hospitals. Data from the National Hospital Discharge Survey. Arch. Intern. Med. 1995, 155, 318-324. [CrossRef]

36. Dai, R.X.; Kong, Q.H.; Mao, B.; Xu, W.; Tao, R.J.; Wang, X.R.; Kong, Q.Y.; Xu, J.F. The mortality risk factor of community acquired pneumonia patients with chronic obstructive pulmonary disease: A retrospective cohort study. BMC Pulm. Med. 2018, 18, 12. [CrossRef] [PubMed]

37. Gravina, G.; Ferrari, F.; Nebbiai, G. The obesity paradox and diabetes. Eat. Weight Disord. 2021, 26, 1057-1068. [CrossRef] [PubMed]

38. Yamauchi, Y.; Yasunaga, H.; Matsui, H.; Hasegawa, W.; Jo, T.; Takami, K.; Fushimi, K.; Nagase, T. Comparison of in-hospital mortality in patients with COPD, asthma and asthma-COPD overlap exacerbations. Respirology 2015, 20, 940-946. [CrossRef] [PubMed]

39. Suissa, S. Inhaled corticosteroids preventing pneumonia mortality: Paradox or selection bias? Eur. Respir. J. 2019, 53, 1802112. [CrossRef]

40. Luna, C.M.; Palma, I.; Niederman, M.S.; Membriani, E.; Giovini, V.; Wiemken, T.L.; Peyrani, P.; Ramirez, J. The impact of age and comorbidities on the mortality of patients of different age groups admitted with community-acquired pneumonia. Ann. Am. Thorac Soc. 2016, 13, 1519-1526. [CrossRef] [PubMed] 
41. Quan, H.; Parsons, G.A.; Ghali, W.A. Validity of procedure codes in International Classification of Diseases, 9th revision, clinical modification administrative data. Med. Care 2004, 42, 801-809. [CrossRef]

42. Akmatov, M.K.; Ermakova, T.; Holstiege, J.; Steffen, A.; von Stillfried, D.; Bätzing, J. Comorbidity profile of patients with concurrent diagnoses of asthma and COPD in Germany. Sci. Rep. 2020, 10, 17945. [CrossRef] [PubMed] 